\title{
Does Drinking Promote Risky Sexual Behavior?
}

\section{A Complex Answer to a Simple Question}

\author{
M. Lynne Cooper \\ University of Missouri-Columbia
}

\begin{abstract}
The present review argues that, popular lore notwithstanding, the well-documented association between usual patterns of alcohol use and risky sex reflects multiple underlying processes that are both causal and noncausal (spurious) in nature. It is further argued that even alcohol's acute causal effects on sexual behavior are more variable than they are commonly assumed to be. Drinking can promote, inhibit, or have no effect on behavior, depending on the interplay of factors governing behavior in a particular situation and the content of individually held beliefs about alcohol's effects.
\end{abstract}

KEYWORDS-alcohol; risky sex; condom use

With the advent of AIDS, efforts to understand the causes of sexual risk-taking have assumed great urgency. In this context, alcohol and its potential disinhibiting effects have received much attention. In the past 20 years, more than 600 studies have been conducted on the link between drinking and risky sex, and drinking proximal to intercourse has become a standard target of intervention efforts aimed at reducing risky sexual behaviors. Targeting drinking as part of a strategy to reduce risky sex can only be effective if drinking causally promotes such behaviors, however. Does the evidence support this connection? Conventional wisdom aside, the answer to this question is surprisingly complex.

\section{BACKGROUND}

The belief that alcohol causally disinhibits sexual behavior is firmly ingrained in our culture. Most people believe that drinking increases the likelihood of sexual activity, enhances sexual experience, and promotes riskier sexual behavior. Many

Address correspondence to M. Lynne Cooper, 105 McAlester Hall, University of Missouri, Columbia, MO, 65211; e-mail: cooperm@missouri. edu. also attribute risky sexual experiences to the fact that they were drinking and report drinking (or plying their partner with alcohol) to exploit alcohol's alleged disinhibiting effects on sexual behavior.

Consistent with popular belief, the overwhelming majority of studies do find an association between the two behaviors (Cooper, 2002; Leigh \& Stall, 1993). The typical study examines the cross-sectional association between usual patterns of drinking and risky sex. For example, in such studies, individuals who drink consistently report more partners than those who abstain do. Owing to design limitations, however, these studies tell us little about the underlying causal relationship. Such data cannot even establish a temporal link between drinking and risky sex, a minimum condition for attributing causality to acute alcohol effects. Thus, although people are quick to infer a causal connection between the two behaviors, multiple interpretations are possible. Three will be considered here.

\section{THIRD-VARIABLE EXPLANATIONS}

Third variable explanations that involve stable (possibly genetically based) features of the individual or of his or her life situation offer one important explanation. For example, a person might both drink and have risky sex to satisfy sensation-seeking needs, because of poor impulse control or coping skills, or in an effort to cope with negative emotions. Consistent with this possibility, Cooper, Wood, Orcutt, and Albino (2003) showed that one third of the statistical overlap (modeled by a higherorder factor) among diverse risk behaviors, including alcohol use and risky sex, could be explained by low impulse control and an avoidant style of coping with negative emotions. Thrill seeking accounted for a much smaller proportion of the overlap, and significantly predicted the overlap only among white (not black) adolescents. In addition, avoidance coping predicted the onset of drinking among initially abstinent youth, and in interaction with impulsivity it predicted the onset of sexual 
behavior among those who were initially virgins. Thus, avoidance coping and impulsivity appear to be important common causes that partially account for the link between drinking and risky sex. Although thrill seeking was not a strong predictor in our randomly constituted, biracial adolescent sample, closely related measures (e.g., sensation seeking) have been shown to fully account for the association between drinking and risky sex in some high-risk samples (e.g., heavy drinkers, gay or bisexual men).

An individual might also drink and have risky sex as part of a lifestyle, such as being single or living in a fraternity house, where both behaviors are tacitly or explicitly encouraged. Consistent with this possibility, perceptions of peer norms related to drinking and sex are among the most robust predictors of involvement in both behaviors among youth. Similarly, characteristics of one's home environment-e.g., living in a singleparent or conflict-ridden household-have also been found to predict both behaviors. Thus, direct evidence showing that covariation between the two behaviors can be explained by third variables, and indirect evidence showing that involvement in both behaviors is linked to the same putative causal factors, support the contention that the association between drinking and risky sex is at least partly due to the influence of underlying common causes.

\section{REVERSE CAUSAL EXPLANATIONS}

Reverse causal explanations posit that the intention or desire to engage in risky sex causes one to drink when sexual opportunity is perceived. Consistent with this possibility, surveys of college students reveal that up to one half of undergraduates report drinking more than usual to make it easier to have sex and giving their partners alcohol to increase the likelihood of sex (Cooper, 2002). Alternatively, an individual might plan a romantic evening and drink to enhance that experience or plan to pick someone up at a party and drink to provide an excuse (to oneself or others) for behavior that might later be seen as inappropriate. Although different motives (to disinhibit, enhance, or excuse) presumably underlie drinking in each scenario, all accounts nevertheless assume that people who drink strategically hold relevant beliefs about alcohol's capacity to facilitate the desired sexual outcome. Supporting this notion, Dermen and I (Dermen \& Cooper, 1994) found that people who believe that alcohol enhances or disinhibits sex are more likely to drink, and to drink to intoxication, in sexual or potentially sexual situations (e.g., on a date). Thus, for at least some people, the intention or desire to have sex may precede and cause drinking, rather than the reverse.

\section{CAUSAL EXPLANATIONS}

Two prominent theories depict alcohol as a cause of disinhibited social behaviors: alcohol myopia and expectancy theories. Al- cohol-myopia theory (Steele \& Josephs, 1990) posits that disinhibited behavior results from an interaction of diminished cognitive capabilities and the specific cues that influence behavior in a given situation. Because alcohol narrows the range of cues perceived and limits the ability to process and extract meaning from these cues, intoxication renders a person susceptible to momentary pressures. Simple, highly salient cues (e.g., sexual arousal) continue to be processed, whereas more distal, complex ones (e.g., fear of pregnancy) are no longer adequately processed. Consequently, alcohol creates a "myopia" in which incompletely processed aspects of immediate experience exert undue influence on behavior and emotion. Accordingly, alcohol has its strongest effect when a behavior is controlled by instigating and inhibiting cues that are strong and nearly equal in force-a circumstance known as inhibition conflict.

In support of this model, Steele and Josephs conducted a meta-analysis (a method for statistically combining effects) of 34 experimental studies testing alcohol's effects on social behavior. Results revealed a small (.14) average standardized effect for alcohol under low-inhibition-conflict conditions versus a large effect (1.06) under high-conflict conditions. Thus, consistent with alcohol-myopia theory, intoxicated participants behaved more extremely than sober ones did primarily under high-conflict conditions.

Whereas alcohol-myopia theory emphasizes pharmacological mechanisms, expectancy theory emphasizes psychological ones. According to this view, an individual's behavior after drinking is driven by pre-existing beliefs (expectancies) about alcohol's effects on behavior, much like a self-fulfilling prophecy (Hull \& Bond, 1986). The role of expectancies has been investigated experimentally in studies that independently manipulate alcohol content and expectancy set (the belief that alcohol has been consumed). In a meta-analysis of 36 such studies, Hull and Bond found that people who believed they had consumed alcohol (but had not) behaved similarly to those who had consumed alcohol (and didn't know it). Indeed, expectancy effects were significant and only slightly smaller than alcohol-content effects (.27 vs. .35). Expectancy theory thus highlights the role of individually held beliefs about alcohol's effects, and suggests by extension that alcohol effects on behavior may vary as a function of these beliefs.

The foregoing indicates that alcohol intoxication can cause more extreme social behavior through both pharmacological and psychological mechanisms. Contrary to popular opinion, these effects are not immutable, but are contingent on the nature of instigating and inhibiting cues governing momentary behavior, on the content of one's beliefs about alcohol effects, or possibly on a combination of both. Theoretically, then, alcohol intoxication should lead to riskier sexual behavior only under certain conditions or among certain people, a contention that existing evidence largely supports. 


\section{A SELECTIVE REVIEW OF NATURALISTIC STUDIES OF DRINKING AND RISKY SEX}

From a public health perspective, one of the most important issues concerns alcohol's potential to facilitate the occurrence of intercourse, especially with new or casual partners. To investigate this issue, Orcutt and I (Cooper \& Orcutt, 1997) examined the link between drinking and intercourse on two first-date occasions in a large, representative sample of adolescents. Although these data are correlational, the within-subjects design allowed us to compare a person's behavior on two occasions that, for many, differed in the presence versus absence of alcohol, thus helping us rule out stable individual differences between drinkers and nondrinkers as an alternative explanation for observed differences in sexual behavior. As Figure 1 illustrates, our results showed that rates of intercourse were higher when the male partner drank and lower when he abstained. Interestingly, however, parallel analyses revealed no such relationship for drinking by the female partner.

Drawing on alcohol-myopia theory, we reasoned that the psychological conditions necessary for alcohol-related disinhibition existed only among men. Specifically, if males experienced a type of conflict in which dominant cues favored behavioral action while peripheral cues favored behavioral inhibition, we would expect (due to the greater difficulty of accessing and processing peripheral cues) alcohol-related disinhibition. In contrast, if females experienced a type of conflict in which dominant cues favored inhibition and peripheral ones activation, then decreased processing of peripheral cues should not disinhibit behavior. Consistent with this logic, we found that men perceived more benefits relative to costs of having sex on their most recent first date, whereas women perceived more costs relative to benefits. Moreover, only the per-

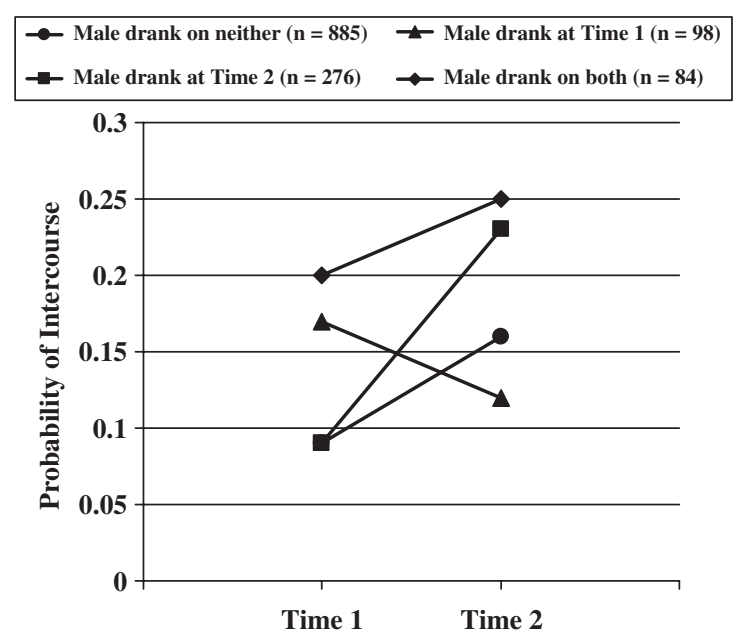

Fig. 1. Male couple-member alcohol use and probability of intercourse on two first-date occasions. From Cooper \& Orcutt (1997).

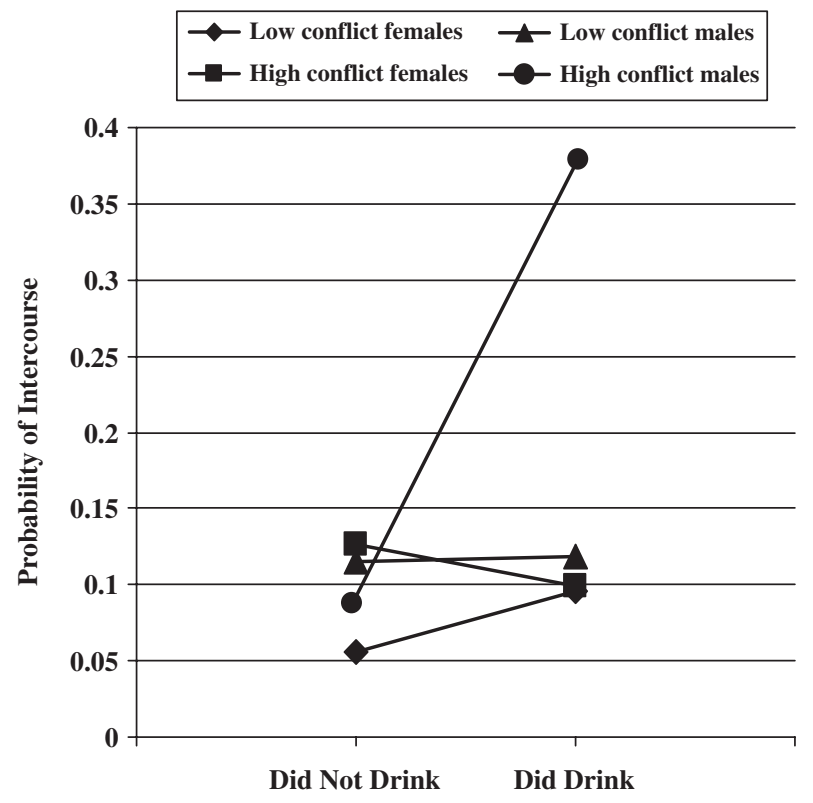

Fig. 2. Alcohol use, gender, and perceived conflict predicting probability of intercourse on the most recent first date. From Cooper \& Orcutt (1997).

ception of increasing costs predicted conflict among men (for whom benefits were more salient), whereas the reverse was true among women. Thus, men and women appeared to experience qualitatively different forms of conflict about having sex on their most recent first date. Moreover, consistent with the idea that the type of conflict conducive to alcohol-related disinhibition occurred only among men, rates of intercourse on the date were significantly elevated only among highly conflicted men who drank alcohol (Fig. 2).

Together these data indicate that how alcohol affects sexual behavior is determined by the content and relative strength of competing cues that inhibit or activate behavior, and they raise the possibility that alcohol might even promote safer behavior under the right circumstances! Recent experimental evidence lends strong support to this idea, showing that when the potential costs of having sex with an attractive new partner were made salient, intoxicated individuals reported more cautious intentions than did sober ones (MacDonald, Fong, Zanna, \& Martineau, 2000).

A second key question from a public health perspective is whether drinking reduces condom use. Somewhat surprisingly, most naturalistic studies directly testing the link between drinking on a specific intercourse occasion and condom use on that occasion find no relationship. Indeed in a quantitative analysis of 29 such tests (Cooper, 2002), alcohol was associated with lower rates of condom (and birth-control) use only under circumscribed conditions: at first intercourse but not on subsequent intercourse occasions, in younger but not older samples, and in studies conducted earlier rather than more recently (Leigh, 2002, reports similar results). 
One plausible interpretation of these findings is that few people experience the type of conflict conducive to alcohol-related disinhibition of condom use, though such conflict may have been common in the past and may still be common among sexually inexperienced, younger adolescents. Although no study has directly tested these ideas, a study conducted by Dermen and me (Dermen \& Cooper, 2000) provides indirect support. We examined feelings of conflict about using a condom on four occasions of intercourse across two different samples (one of college students; one of community-residing young adults, aged 19-25), and found that fewer than $15 \%$ of participants were highly conflicted about using a condom on each occasion. Moreover, although drinking did not predict lower overall rates of condom use on any of these occasions, it predicted significantly lower rates (in three of four tests) among those who felt conflicted about using a condom on that occasion.

In short, these data suggest that drinking can undermine safe sex behaviors, but that it does not invariably do so. Rather, alcohol can promote, inhibit, or have no effect on risky sexual behaviors depending on the specific constellation of salient cues in the moment.

\section{THE ROLE OF ALCOHOL EXPECTANCIES}

Although the preponderance of evidence suggests that inhibition conflict plays the larger role in accounting for alcohol's acute causal effects on risky sexual behavior, expectancies also appear important. As previously discussed, those who believe that alcohol disinhibits or enhances sexual experience are more likely to drink in (potentially) sexual situations, suggesting that expectancies are instrumental in setting up situations that may lead to alcohol-related disinhibition of sex. Expectancies (in the absence of alcohol) have also been shown to influence other aspects of sexual experience that could indirectly promote risky behaviors. For example, a recently conducted experiment in which participants were paired with previously unknown, opposite-sex partners found that participants who thought they had consumed alcohol (though none had been consumed) reported greater sexual arousal, perceived their partners as more sexually disinhibited, and showed erotic slides (presumed to be a behavioral analog of sexual interest) to their partners significantly longer, but only if they also held strong beliefs about alcohol's capacity to disinhibit or enhance sexual experience (George, Stoner, Norris, Lopez, \& Lehman, 2000). These data suggest that expectancies, once activated by alcohol consumption, may strengthen instigating cues for sex, thereby bringing an individual for whom costs might otherwise greatly outweigh benefits into a state of high inhibition conflict. Finally, expectancies have also been shown to interact with feelings of conflict to jointly predict alcohol-related disinhibition of risky sexual behavior (Dermen \& Cooper, 2000). Thus, expectancies and actual alcohol content might work in tandem to disinhibit risky sexual behavior in real-world situations where the two processes always co-occur.

\section{CONCLUSIONS AND FUTURE DIRECTIONS}

The relationship between alcohol use and risky sex is complex. It cannot be explained by a single mechanism, but instead reflects multiple underlying causal and noncausal processes. Moreover, even the causal portion of this relationship is not manifest as a main effect but as an interaction.

These complexities have important implications for both research and intervention efforts. The multiplicity of plausible causal mechanisms highlights the need for diverse methodological approaches for exploring alternative models, and for greater sophistication in framing research questions. Rather than focusing on which model better accounts for the link between drinking and risky sex, future research should focus on delineating the conditions under which, and the individuals for whom, different causal (and noncausal) processes are most likely to operate.

At the same time, researchers trying to unravel alcohol's acute effects must adopt more sophisticated methods for studying the complex interplay between drinking, individually held expectancies, and situational cues. Diary methods in which people report on both behaviors across multiple days provide an important and ecologically valid approach for examining this relationship. Such methods not only enable more accurate assessment of the behaviors themselves but also provide a window onto the motivations, emotions, and cognitions that subtly shape these behaviors and set the stage for alcohol's variable effects across individuals and situations.

The existence of multiple causal models also points to the need for diverse intervention strategies, and raises the possibility that different strategies will be optimally effective among individuals for whom different causal processes dominate. For example, among people who chronically drink and engage in risky behaviors, the relationship between drinking and risky sex may primarily reflect the influence of underlying common causes. For such individuals, universal change strategies targeting these common causes should be maximally efficacious. Alternatively, carefully designed interventions aimed at reducing drinking (or manipulating risk cues) in settings where drinking and encountering potential partners co-occur (e.g., college bars) could lower sexual risks associated with alcohol use among those who are most vulnerable to acute intoxication effects, situational influences, or both. To be maximally effective, interventions must be carefully tailored for different populations and circumstances in which different underlying causal processes predominate.

\section{Recommended Reading}

Cooper, M.L. (2002). (See References)

George, W.H., \& Stoner, S.A. (2000). Understanding acute alcohol effects on sexual behavior. Annual Review of Sex Research, 11, 92122 . 
Leigh, B.C., \& Stall, R. (1993). (See References)

Weinhardt, L.S., \& Carey, M.P. (2000). Does alcohol lead to sexual risk behavior? Findings from event-level research. Annual Review of Sex Research, 11, 125-157.

\section{REFERENCES}

Cooper, M.L. (2002). Alcohol use and risky sexual behavior among college students and youth. Journal of Studies on Alcohol, 14(Suppl.), 101-117.

Cooper, M.L., \& Orcutt, H.K. (1997). Drinking and sexual experiences on first dates among adolescents. Journal of Abnormal Psychology, 106, 191-202.

Cooper, M.L., Wood, P.K., Orcutt, H.K., \& Albino, A.W. (2003). Personality and predisposition to engage in risky or problem behaviors during adolescence. Journal of Personality and Social Psychology, 84, 390-410.

Dermen, K.H., \& Cooper, M.L. (1994). Sex-related alcohol expectancies among adolescents. Psychology of Addictive Behaviors, 8, $161-168$.
Dermen, K.H., \& Cooper, M.L. (2000). Inhibition conflict and alcohol expectancy as moderators of alcohol's relationship to condom use. Experimental and Clinical Psychopharmacology, 8, 198-206.

George, W.H., Stoner, S.A., Norris, J., Lopez, P.A., \& Lehman, G.L. (2000). Alcohol expectancies and sexuality: A self-fulfilling prophecy analysis of dyadic perceptions and behavior. Journal of Studies on Alcohol, 61, 168-176.

Hull, J.G., \& Bond, C.F. (1986). Social and behavioral consequences of alcohol consumption and expectancy: A meta-analysis. Psychological Bulletin, 99, 347-360.

Leigh, B.C. (2002). Alcohol and condom use: A meta-analysis of eventlevel studies. Sexually Transmitted Disease, 29, 476-482.

Leigh, B.C., \& Stall, R. (1993). Substance use and risky sexual behavior for exposure to HIV: Issues in methodology. American Psychologist, 48, 1035-1045.

MacDonald, T.K., Fong, G.T., Zanna, M.P., \& Martineau, A.M. (2000). Alcohol myopia and condom use: Can alcohol intoxication be associated with more prudent behavior? Journal of Personality and Social Psychology, 78, 605-619.

Steele, C.M., \& Josephs, R.A. (1990). Alcohol myopia: Its prized and dangerous effects. American Psychologist, 45, 921-932. 